\title{
Morfometría Nuclear de Hepatocitos Esteatósicos y Esteatósicos Estimulados con Laser Infrarrojo
}

\author{
Nuclear Morphometric of the Steatotic Hepatocytes and Stimulated Steatotisc with Infrared Laser
}

\author{
R. Cornejo; O. Garrido*** \& R. Jaramillo**
}

CORNEJO, R.; GARRIDO, O. \& JARAMILLO, R. Morfometría nuclear de hepatocitos esteatosicos y esteatosicos estimulados con laser infrarrojo. Int. J. Morphol., 34(1):375-379, 2016.

RESUMEN: Un total de 24 ratas hembras de 4 meses de vida con peso aproximado de 250 g recibieron una solución de alcohol $40 \%$ disuelto en agua lo cual derivó en una esteatosis alcohólica multivesicular. A 12 de estas ratas esteatósicas se le aplicó estimulaciones de láser infrarrojo con dosis de $8 \mathrm{~J} / \mathrm{cm}^{2}$ durante 15 días consecutivos. Posteriormente las ratas fueron sacrificadas y se extrajeron muestras tanto de hígado esteatósico como del estimulado para enseguida ser procesadas para microscopía electrónica de transmisión. De hepatocitos esteatósicos y esteatósicos estimulados se obtuvieron microfotografías electrónicas de transmisión con aumentos finales de 9.500 X, las cuales fueron sometidas a estudios morfométricos para determinar tanto el número de poros nucleares como de fracciones volumétricas de los siguientes componentes celulares: Areas celular y nuclear, fracciones volumétricas de núcleos y nucléolos, eu y heterocromatina. De igual manera se determinó la relación nucleo-citoplasmática de ambos tipos celulares. Del análisis de los resultados entre hepatocitos alcohólicos y alcohólicos estimulados se visualiza que existen notables diferencias en todos los componentes celulares cuantificados. Se concluye que los efectos de la estimulación con lásr infrarrojo provoca en los hepatocitos una drástica transformación en su ultraestructura y en su morfología, situación que se traduciría, por ende, en una variación funcional, representando de esta manera el efecto que dicha estimulación provoca en los hepatocitos.

PALABRAS CLAVE: Esteatosis; Láser; Morfometría; Hepatocito.

\section{INTRODUCCIÓN}

La patología referida como hígado graso es la más frecuente de las afecciones conocidas como esteatosis, está caracterizada por la deposición anormal de lípidos tanto en los espacios interticiales del hígado como en los citoplasmas de los hepatocitos, revelando anormalidad en los procesos de síntesis y eliminación de triglicéridos (Ishak et al., 1991).

Dichos lípidos se acumulan formando vesículas que cuando son de mayor tamaño rodean al núcleo desplazándolo hacia la periferia celular pegado a la membrana plasmática, generando así la denominada esteatosis macrovesicular. Por el contrario, si el lípido se estructura en pequeñas gotas ocupando distintas áreas del citoplasma se produce la esteatosis microvesicular (Arakawa et al., 1985).

Se ha demostrado que la etiología de la esteatosis se relaciona con componentes metabólicos y nutricionales (Alpers \& Isselbacher, 1975), farmacológicos generalmente provocado por antibióticos y mayoritariamente con un consumo exacerbado de toxinas como etanol y tetracloruro de carbono (Day \& Yeaman, 1994), existiendo también diversos factores de riesgo asociado a la afección tales como diabetes mellitus (Araya et al., 2006), mal nutrición proteica (Conde Martel et al., 1993), hipertensión (Brokes \& Cooper, 2007), obesidad (Saadeh, 2007) y anoxia (Cotran et al., 1998). De igual manera Day et al. (1993) sugieren intervención de factores genéticos en el desarrollo de esta patología.

Así mismo, la expresión alterada en los hepatocitos de proteínas/enzimas involucradas en la oxidación de ácidos grasos así como de las constitutivas de peroxisomas, se traduciría en la acumulación de triglicéridos hepáticos (Rehnmark et al., 1998). Por otra parte, modificaciones en la morfología tanto de la matríz como el aumento en los diámetros de la membrana interna mitocondrial, sugieren disminución en los mecanismos de beta oxidación de ácidos grasos y generación de esteatosis microvesicular (Santer et al., 1990).

\footnotetext{
* Departamento de Ciencias Básicas, Universidad de La Frontera, Temuco, Chile.

${ }^{* *}$ Instituto de Ciencias Marinas y Limnológicas, Universidad Austral de Chile, Valdivia, Chile.

Financiado por Proyecto DIUFRO 15-0005.
} 
Es objetivo fundamental del presente trabajo visualizar las diferencias morfológicas, de volumen y de número que se puedan establecer mediante la microscopía electrónica de transmisión y técnicas morfométricas, comparando tanto las áreas y fracciones volumétricas de estructuras nucleares como de número de poros nucleares de hepatocitos esteatósicos y esteatósicos estimulados con LIR. De igual manera se cuantificó la relación núcleo-citoplasmática y celular de ambos hepatocitos.

En la medida que se cuantifiquen y comparen a nivel ultraestructural estos parámetros en estos tipos celulares, se podria determinar las variaciones en la funcionalidad que adquieren sobre todo aquellos que contienen las infiltraciones lipídicas.

\section{MATERIAL Y MÉTODO}

Un total de 24 ratas hembras de 4 meses de vida con peso aproximado de $250 \mathrm{~g}$ recibieron una solución de alcohol $40 \%$ disuelto en agua lo cual derivó en una esteatosis alcohólica multivesicular. A 12 de estas ratas esteatósicas se le aplicó estimulaciones de láser infrarrojo con dosis de $8 \mathrm{~J} /$ $\mathrm{cm}^{2}$ durante $15 \mathrm{~d}$ consecutivos.

Posteriormente las ratas fueron sacrificadas y se extrajeron muestras tanto de hígado esteatósico como del esteatósico estimulado con LIR para enseguida ser procesadas para microscopía electrónica de transmisión donde a las muestras se le adicionó una solución de glutaraldehído 2 $\%$ en tampón fosfato $0,15 \mathrm{M}, \mathrm{pH} 7,2$ y se mantuvo a temperatura ambiente por $2 \mathrm{~h}$. Luego, fueron sometidas a un lavado en solución de $6 \mathrm{~g}$ de $\mathrm{NaCl}$ y $73 \mathrm{~g}$ de sacarosa, disuelto en un litro de agua destilada.

La post-fijación se realizó con tetróxido de osmio $1 \%$ disuelto en la solución antes descrita durante una hora a $40{ }^{\circ} \mathrm{C}$ y acetato de uranilo $0,5 \%$ por $18 \mathrm{~h}$. Luego de lavado el material fue deshidratado en concentraciones crecientes de acetona (30 a 100\%) e incluido en Araldita 6005. Se obtuvieron cortes ultrafinos de aproximadamente $70 \mathrm{~nm}$ de grosor los que fueron tratados con acetato de uranilo $2 \%$ durante 40 min y citrato de plomo $0,5 \%$ por $10 \mathrm{~min}$.

Las muestras fueron finalmente estudiadas y microfotografiadas en un microscopio electrónico Phillips EM 300.

Análisis estadístico. A partir de los bloques para microscopía electrónica fueron obtenidos cortes ultrafinos desde los cua- les se micrografiaron hepatocitos irradiados con distintas dosis con un aumento final de hasta $9.500 \mathrm{X}$.

Para la evaluación de las fracciones volumétricas de los componentes celulares, fue sobrepuesto un retículo de puntos sobre las microfotografías electrónicas y se procedió al conteo diferencial de los puntos que incidían sobre los perfiles de los componentes, calculándose la fracción volumétrica que ellos ocupan, mediante la siguiente ecuación (Weibel, 1969), donde:

$$
F v=\frac{P i}{P t}
$$

$\mathrm{Fv}=$ Fracción volumétrica del componente celular.

$\mathrm{Pi}=$ Puntos incidentes sobre el componente en estudio.

$\mathrm{Pt}=$ Puntos totales incidentes en la célula estudiada.

Todos los datos cuantitativos obtenidos fueron sometidos a la prueba de Wilcoxon para muestras no paramétricas estableciendo diferencias con un valor de $\mathrm{Z}=2,023$ y un $\mathrm{p}=$ 0,043 indicando entonces que existen diferencias significativas entre ellos.

\section{RESULTADOS}

La microscopía electrónica de transmisión utilizada en nuestro estudio muestra la morfología de hepatocitos afectados con esteatosis microvesicular y de los esteatósicos estimulados con LIR, detallándose en ellos la ultraestructura de sus componentes nucleares, evidenciados en las Figuras 1 y 2 respectivamente y que generan los siguientes datos morfométricos los cuales se consignan en las Tablas I, II y III.

Tabla I. Número de poros nucleares cuantificados unidireccionalmente en hepatocitos de rata esteatósicos y esteatósicos estimulados con LIR.

\begin{tabular}{lc}
\hline Hepatocito & Número Poros Nucleares \\
\hline Esteatósico & 15 \\
Esteatósico Lir & 35 \\
\hline
\end{tabular}

Tabla II. Áreas celulares y nucleares expresadas en $\mathrm{m}^{2}$ pertenecientes tanto a hepatocitos de rata esteatósicos. y esteatósicos estimulados con LIR.

\begin{tabular}{lcc}
\hline & $\begin{array}{c}\text { Hepatocito } \\
\text { Esteatósico }\end{array}$ & $\begin{array}{c}\text { Hepatocito } \\
\text { estimulado LIR }\end{array}$ \\
\cline { 2 - 3 } Área Celular & 249 & 434 \\
Área Nuclear & 43 & 55 \\
\hline
\end{tabular}




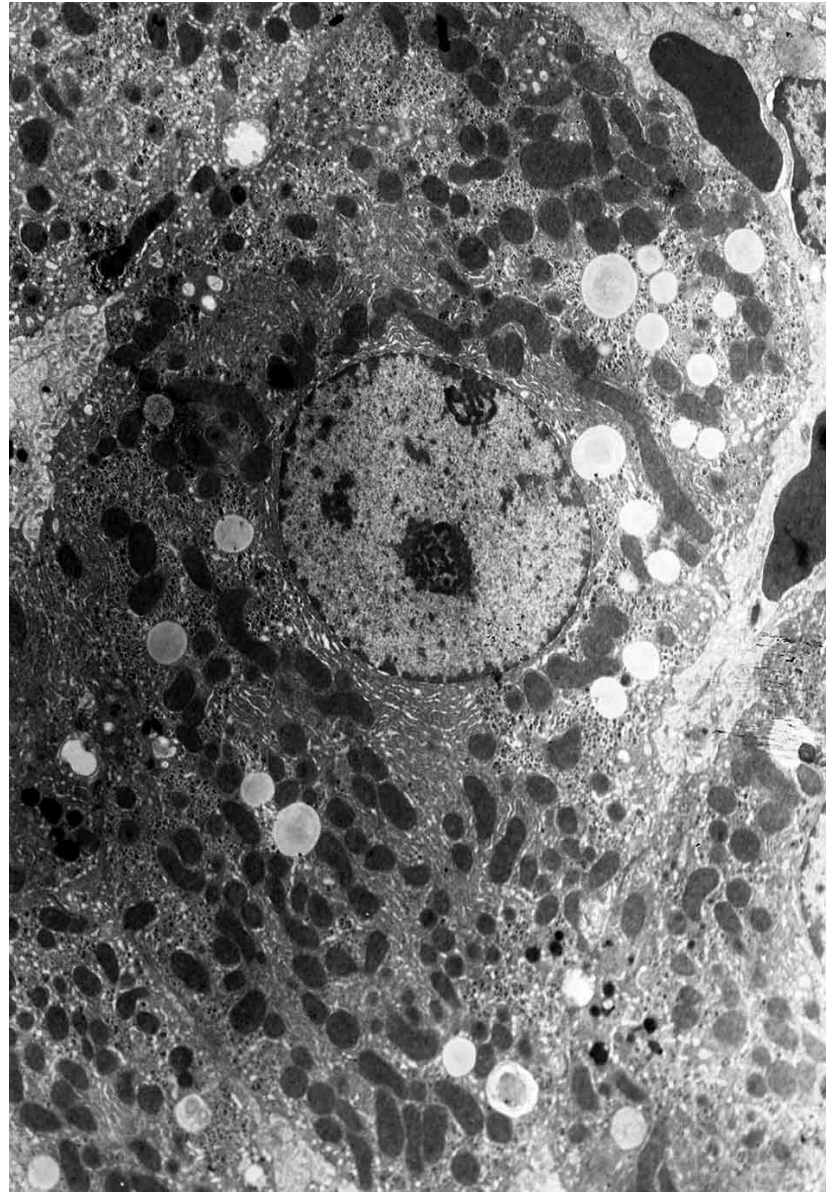

Fig. 1. Microfotografía electrónica que muestra la morfología ultraestructural de los componentes nucleares de hepatocitos esteatósicos estimulados con láser infrarrojo (LIR).

Tabla III. Fracciones volumétricas expresadas en porcentaje correspondiente tanto a eu y heterocromatina como nucleólos evaluadas en hepatocitos de rata esteatósicos y esteatósicos estimulados con LIR

\begin{tabular}{lcc}
\hline & $\begin{array}{c}\text { Hepatocito } \\
\text { esteatósico }\end{array}$ & $\begin{array}{c}\text { Hepatocito } \\
\text { estimulado LIR }\end{array}$ \\
\cline { 2 - 3 } Heterocromatina & $8 \%$ & $27 \%$ \\
Eucromatina & $92 \%$ & $73 \%$ \\
Nucléolo & $16 \%$ & $10 \%$ \\
\hline
\end{tabular}

\section{DISCUSIÓN}

El análisis de los resultados obtenidos evidencia que el efecto de las emisiones infrarrojas sobre hepatocitos es capaz de generar notables cambios en su ultraestructura y por ende en su funcionalidad. En este contexto se visualiza claramente que producto de las citadas estimulaciones se genera un aumento en el área nuclear de los hepatocitos que equivale a $12 \mathrm{~m}^{2}$.

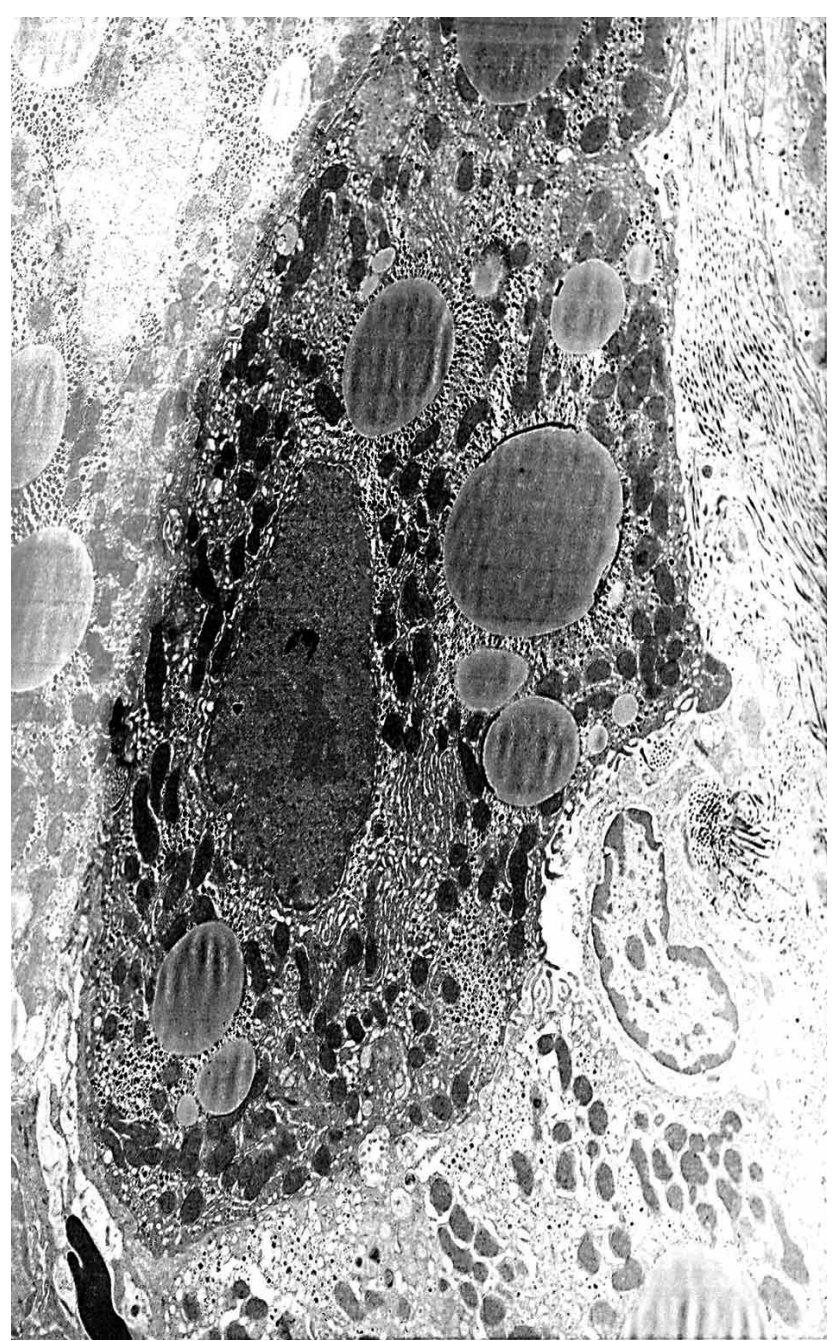

Fig. 2. Microfotografía electrónica que muestra la morfología ultraestructural de los componentes nucleares de hepatocitos afectados con esteatosis microvesicular.

Este hallazgo había sido ya demostrado pero solo producto de la microscopía óptica por Cornejo et al., (2010) en esa oportunidad intentado precisar las dosis óptimas para lograr una mayor actividad celular.

De igual manera, este resultado coincide con los obtenidos por Zhang et al. (2003) cuando irradiaba con LIR fibroblastos humanos resultando en la expresión de numerosos genes que regulan importantes funciones celulares como la proliferación, la apoptosis, las propiedades antioxidantes y el metabolismo energético.

En este mismo sentido, los estudios de EhrenhoferMurray (2004) precisan que las estimulaciones infrarrojas provocan cambios determinantes en la estructura de la cromatina, especialmente en la eucromatina, situación que 
desencadena hiperplasia de los componentes nucleares y una elevada actividad celular.

Claramente existe un notable incremento en el área celular de los hepatocitos estimulados que corresponde a $185 \mathrm{~m}^{2}$ aumento que podría ser generado producto de procesos de síntesis citoplasmática como de mecanismos de transporte de sustancias hacia el citoplasma que provocarían un incremento de la masa citosólica y por ende un mayor volumen celular (Lodish et al., 2002).

En la perspectiva del análisis cuantitativo de los poros nucleares evidentemente se observa una gran variación en los hepatocitos estimulados con respecto a los sin activación, donde se duplica el número de ellos, situación que de alguna forma ilustra relativo a un funcionamiento desarrollado de transporte núcleocitoplasmático intenso, este resultado nuestro se relaciona positivamente con los comunicados por Schroeder $e t$ al. (2007) quienes en estudios sobre efectos de LIR en fibroblastos describe una gran actividad en el transporte entre citoplasma ynúcleo lo que se traduce en una activación en la expresión y transcripción génica para la síntesis de metaloproteasas, proteína componente de la matriz extracelular.

Relacionando este último resultado con los anteriormente señalados nuestro resultado igualmente es coincidente con lo expresado por Paniagua et al. (2007) los cuales plantean que naturalmente la cantidad de poros de una determinada célula está relacionada directamente con su funcionalidad, es decir, a mayor función celular presencia de mayor número de poros nucleares.

Finalmente, con respecto a los volúmenes tanto de cromatinas como de nucléolos cuantificados relacionando ambos tipos de hepatocitos nuestros datos son semejantes a los logrados por Hernandez-Verdum (2010) siendo posible concluir que ambas células poseen porcentajes acorde tanto a su función celular de expresión génica como en el caso de nucléolos a la biosíntesis ribosomal para la generación de proteínas propias de estas células en diferente estadio funcional.

Podemos finalmente concluir que los resultados aquí presentados y su correspondiente discusión, definen con claridad las características morfométricas y ultraestructurales de las inducciones infrarrojas sobre hepatocitos esteatósicos en comparación con aquellos sin estimular, destacando que se han generado diferencias significativas en el desarrollo de estructuras nucleares y que, por ende, se incrementa la actividad celular en los hepatocitos activados con el LIR.
CORNEJO, R.; GARRIDO, O. \& JARAMILLO, R. Nuclear morphometric of the steatotic hepatocytes and stimulated steatotisc with infrared laser. Int. J. Morphol., 34(1):371-374, 2016.

SUMMARY: A total of 24 female rats, aged 4 months and weighing approximately $250 \mathrm{~g}$ and they given a solution of $40 \%$ alcohol dissolved in water, leading to alcoholic multivesicular steatosis and 12 of rats was given and infrared laser with dose of 8 $\mathrm{J} / \mathrm{cm}^{2}$ during $15 \mathrm{~d}$. The rats were then killed and samples of steatosis and stimulated and were taken and processed for examination by transmission electron microscope. Transmission electron microscope microphotographs steatotic hepatocytes and stimulated steatotic were obtained with final magnification of 9,500 X. They were subjected to morphometric studies to determine the number of nuclear pores and volumetric fractions and areas the following components: cellular and nuclear area, volumetric fractions of nucleus, nucleolus, eu and heterochromatin, nucleocytoplamic ratio of each cell type was determined. Analysis of the results between alcoholic hepatocytes and stimulate alcoholic shows that noticeable differences exist in all the cell components quantified. It is concluded that the effects of the stimuli of laser infrared provoke in the hepatocytes, a drastic transformation of their ultrastructure and morphology. This finally leads to functional variations, representing the effects produced by this stimulate in the hepatocytes.

KEY WORDS: Steatosis; Laser; Morphometry; Hepatocytes.

\section{REFERENCIAS BIBLIOGRÁFICAS}

Alpers, D. H. \& Isselbacher, K. J. Fatty Liver: Biochemical and Clinical Aspects. In: Schiff, L. (Ed.). Diseases of the Liver. Philadelphia, Lippincott, 1975. pp.815-32.

Arakawa, M.; Taketomi, S.; Furuno, K.; Matsuo, T. \& Iwatsuka, H. Metabolic studies on the development of ethanol-induced fatty liver in KK-Ay mice. J. Nutr., 105(12):1500-8, 1975.

Araya, Q. A. V.; Valera, M. J. M.; Contreras, B. J.; Csendes, J. A.; Díaz, J. J. C.; Burdiles, P. P.; Rojas, C. J.; Maluenda, G. F.; Smok, S. G. \& Poniachik, T. J. Glucose tolerance alterations and frequency of metabolic syndrome among patients with non alcoholic fatty liver disease. Rev. Med. Chil., 134(9):1092-8, 2006.

Brookes, M. J. \& Cooper, B. T. Hypertension and fatty liver: guilty by association? J. Hum. Hypertens., 21(4):264-70, 2007.

Conde Martel, A.; González Reimers, E.; Santolaria Fernández, F.; Castro Alemán, V.; Marchena Gómez, J. \& Martínez Riera, A. Liver changes in protein malnutrition. An experimental study in rats. Nutr. Hosp., 8(6):358-63, 1993.

Cornejo, U. R.; Matamala, V. F.; Silva, M. H.; Garrido, O. O.; Buchegger, M. K. \& Jarpa, H. A. Morphometric analysis of 
the effect of infrared laser stimulation on hepatocyte nuclei in rats. Int. J. Morphol., 28(3):771-5, 2010.

Cotran, R.; Kumar, V. \& Robbins, S. Patologia Estrutural e Funcional. $4^{a}$ ed. Río Janeiro, Guanabara-Kogan, 1991. p.150.

Day, C. P.; James, O. F.; Brown, A. S.; Bennett, M. K.; Fleming, I. N. \& Yeaman, S. J. The activity of the metabolic form of hepatic phosphatidate phosphohydrolase correlates with the severity of alcoholic fatty liver in human beings. Hepatology, 18(4):8328, 1993.

Day, C. P. \& Yeaman, S. J. The biochemistry of alcohol-induced fatty liver. Biochim. Biophys. Acta, 1215(1-2):33-48, 1994.

Ehrenhofer-Murray, A. E. Chromatin dynamics at DNA replication, transcription and repair. Eur. J. Biochem., 271(12):2335-49, 2004.

Hernandez-Verdum, D.; Roussel, P.; Thiry, M.; Sirri, V. \& Lafontaine, D. L. The nucleolus: structure/function relationship in RNA metabolism. Wiley Interdiscip. Rev. RNA, 1(3):41531,2010

Ishak, K. G.; Zimmerman, H. J. \& Ray, M. B. Alcoholic liver disease: pathologic, pathogenetic and clinical aspects. Alcohol. Clin. Exp. Res., 15(1):45-66, 1991.

Lodish, H.; Berk, A.; Zipursky, S. L.; Matsudaira, P.; Baltimore, D. \& Darnell, J. Biologia Celular y Molecular. Buenos Aires, Médica Panamericana 2002. pp.579-612.

Paniagua, R.; Nistal, M.; Sesma, P.; Alvarez-Uría, M.; Fraile, B.: Anadón, R. \& Sáez, F. J. Biología Celular. $3^{\mathrm{a}}$ ed. Madrid, McGraw-Hill / Interamericana, 2007.

Rehnmark, S.; Giometti, C. S.; Slavin, B. G.; Doolittle, M. H. \& Reue, K.. The fatty liver dystrophy mutant mouse: microvesicular steatosis associated with altered expression levels of peroxisome proliferator-regulated proteins. J. Lipid Res., 39(11):2209-17, 1998.

Saadeh, S. Nonalcoholic Fatty liver disease and obesity. Nutr. Clin. Pract., 22(1):1-10, 2007.

Santer, R.; Schmidt-Sommerfeld, E.; Leung, Y. K.; Fischer, J. E. \& Lebenthal, E. Medium-chain acyl CoA dehydrogenase deficiency: electron microscopic differentiation from Reye syndrome. Eur. J. Pediatr., 150(2):111-4, 1990.

Schroeder, P.; Pohl, C.; Calles, C.; Marks, C.; Wild, S. \& Krutmann, J. Cellular response to infrared radiation involves retrograde mitochondrial signaling. Free Radic. Biol. Med., 43(1):12835, 2007.

Zhang, Y.; Song, S.; Fong, C. C.; Tsang, C. H.; Yang, Z. \& Yang, M. cDNA microarray analysis of gene expression profiles in human fibroblast cells irradiated with red light. J. Invest. Dermatol., 120(5):849-57, 2003.
Weibel, E. R. Stereological principles for morphometry in electron microscopic cytology. Int. Rev. Cytol., 26:235-302, 1969.

\author{
Dirección para Correspondencia: \\ Dr. Ricardo Cornejo \\ Facultad de Medicina \\ Universidad de La Frontera \\ Casilla 54-D \\ Temuco \\ CHILE
}

Email: rene.cornejo@ufrontera.cl

Received: 18-11-2015

Accepted: 27-12-2016 